\title{
Energy Drink Induced Ventricular Fibrillation and Cardiac Arrest: A Successful Outcome
}

\author{
Rafay Khan a, b, Mohamed Osman ${ }^{\text {a }}$, Shoaib Zafara, Shuvendu Sen ${ }^{\mathrm{a}}$
}

\begin{abstract}
The use of energy drinks, especially among students, has increased exponentially over the past several years. In those with excess consumption, there is a risk of medical consequences. In patients with premature conditions, especially that pertaining to the cardiovascular system, it remains prudent that advisory cautions be appropriately taken. Few cases have demonstrated a correlation of energy drink use and cardiopulmonary arrest but the method of which it can impact certain populations over others has yet to be fully analyzed. In this case report, we discuss the effects of energy drinks on a 27 -year-old male with a history of premature ventricular complexes (PVCs) resulting in cardiopulmonary arrest. Whether there is a link between energy drink consumption and prior risk factors of cardiac deterioration has yet to be fully researched. In this report, we will demonstrate whether there is an association of PVCs, QT prolongation, and cardiac pulmonary arrest with energy drink use.
\end{abstract}

Keywords: Energy drink; Cardiac arrest; Ventricular fibrillation; Arrhythmia

\section{Introduction}

Although there is no standard definition which incorporates energy drinks, the ingredients mostly consist of a non-alcoholic beverage containing caffeine, taurine, vitamins, and some combination of other ingredients used to improve performance and energy which was first introduced in Japan in the 1960s [1]. Adverse events associated with its consumption are shown to be associated with the excess intake of caffeine resulting in sympathomimetic effects. Several reports have illustrated the

Manuscript accepted for publication August 13, 2015

anternal Medicine Department, Raritan Bay Medical Center, 530 New Brunswick Avenue, Perth Amboy, NJ 07733, USA

${ }^{b}$ Corresponding Author: Rafay Khan, Internal Medicine Department, Raritan Bay Medical Center, 530 New Brunswick Avenue, Perth Amboy, NJ 07733, USA. Email: rafay.t.khan@gmail.com

doi: http://dx.doi.org/10.14740/jmc2259w impact of these beverages on the cardiac system which have resulted in cardiac arrest. However, in this presentation, we will demonstrate whether there is a possibility that energy drinks can spark ventricular fibrillation in those with previous cardiac anomalies such as premature ventricular complexes (PVCs), QT prolongation, and a family history of cardiac conditions.

\section{Case Report}

A 27-year-old male with a history of PVCs in the past was brought to the hospital by ambulance after cardiopulmonary arrest. As per the patient's friends, while driving, they noticed the patient had become very rigid and started experiencing convulsions. The patient soon after became unresponsive and was found to be pulseless. They stopped the car and initiated cardiopulmonary resuscitation (CPR). When emergency medical services (EMS) arrived, the patient's friends were still conducting CPR; however, the patient was at this time found to be in ventricular fibrillation. The patient was shocked two times. CPR was continued for $15 \mathrm{~min}$ at which point there was a return of spontaneous circulation; however, he remained comatose and encephalopathic. The patient was intubated in the field and brought to the emergency department. No flow time was less than 2 min and down time was 15 min.

The day prior, the patient's friends stated that he was in a normal state of health. Per his friends, the patient does smoke marijuana on occasion but last use was a week prior. However, they stated although the patient does not have any other history of drug abuse they state he drinks six red bull energy drinks per day. His ongoing red bull consumption has been almost daily for over 6 - 8 months. The patient is currently studying to be a physician assistant and uses the energy drink to help him concentrate with his studies. Past medical history is only notable for history of PVCs noted a few years prior. There is also a strong family history of premature coronary artery disease in the patient's father and grandfather.

On physical examination, the patient was already intubated. His vital signs showed a blood pressure of 120/82 mm $\mathrm{Hg}$, pulse of 97 , respirations of 20 , and temperature of $97.4^{\circ} \mathrm{F}$. Patient was actively moving all his limbs but was not responding to voice or painful stimuli. Patient's eyes were open but he did not follow or track with his eyes. Lung examination did show some scattered rhonchi. Glasgow coma scale was evalu- 


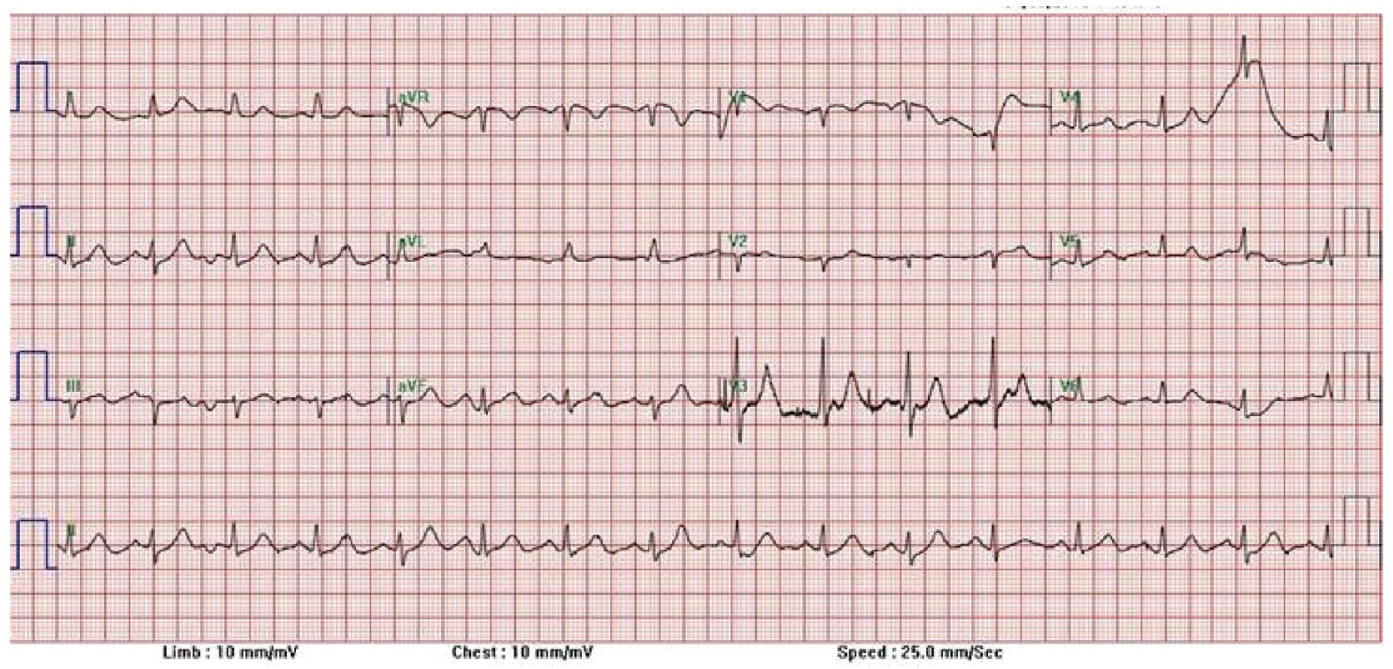

Figure 1. The 12-lead EKG at initial presentation, showing borderline QT prolongation with a QT interval of 469.

ated to be 6. Laboratory data showed hemoglobin of 15.4 , hematocrit of 47, white blood cell count of 14.2, and platelets of 213. Sodium was 140 , potassium 2.8 , chloride 99 , bicarbonate level 21, blood urea nitrogen 15 , creatinine 1.4 , and glucose 205. Alcohol level was negative. Troponin was negative. BNP was measured at 40.84 and lactic acid was elevated at 5.9. Patient's initial urine drug screen was positive for cannabinoids. Arterial blood gas showed a $\mathrm{pH}$ of 7.16, $\mathrm{pCO}_{2}$ of $9, \mathrm{pO}_{2}$ of 79 , and bicarbonate of 21 on $\mathrm{FiO}_{2}$ of $100 \%$. Chest X-ray and head CT scan without contrast were both within normal limits. EKG was done which showed sinus rhythm with borderline QT prolongation and non-specific ST-T wave changes (Fig. 1). Patient had a QTc of 469, which could be attributed to his hypokalemia; however, a congenital prolonged QT could not be excluded.

The patient was admitted to the critical care unit. As per cardiology, he was started on amiodarone and for antibiotic coverage he received one dose of vancomycin and was placed on zosyn. As the patient remained unresponsive, hypothermia protocol was initiated. Target temperature modulation was initiated and the patient was cooled overnight, subsequently in the morning upon nursing evaluation he was noted to be moving and awake. Sedation was discontinued and the patient was awake and following commands. The targeted temperature modulation at that time was terminated. Repeat laboratory work was significant for an elevated CK level from 173 to 3,805. MB fraction increased from 1.14 to 10.7 and tropoinin was elevated at 0.4 likely secondary to cardiopulmonary resuscitation.

Echocardiogram showed questionable hypokinesia and due to his family history and borderline ST elevations on lateral leads on repeat EKG (Fig. 2), cardiac catheterization was planned. The patient underwent cardiac catheterization which showed angiographically normal coronary arteries and a re-

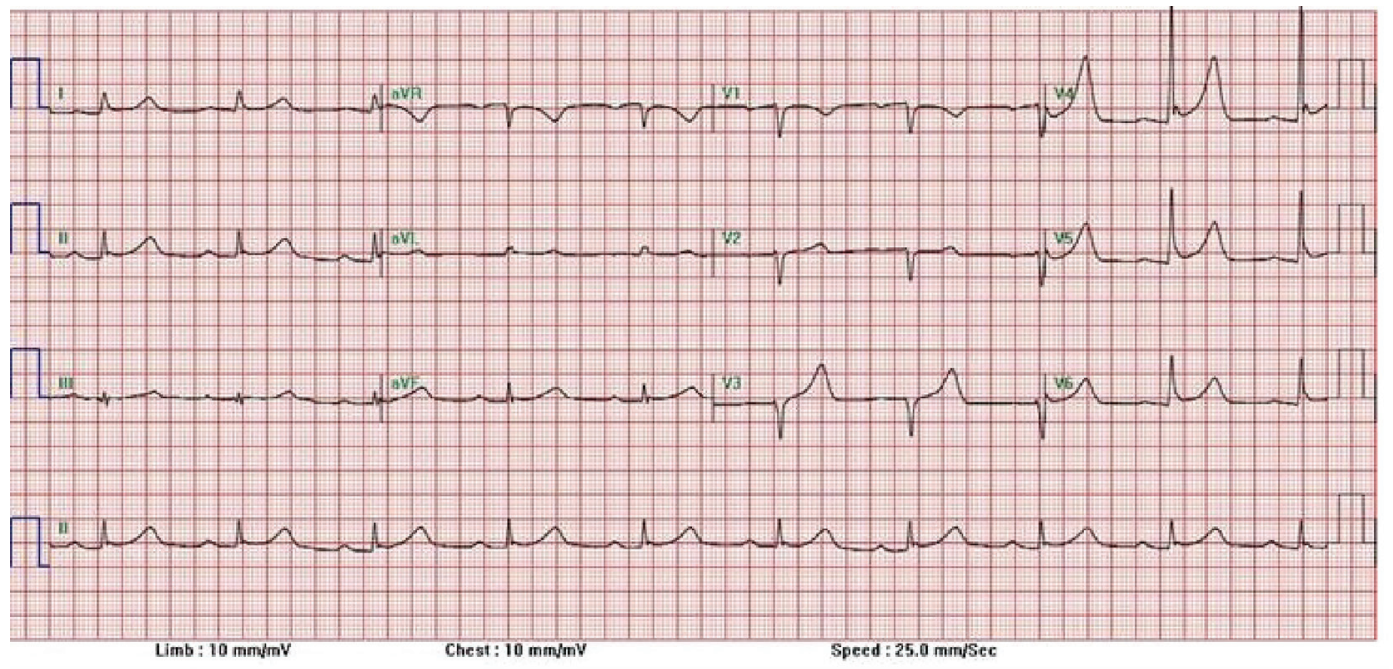

Figure 2. Repeat 12-lead EKG post hypothermia protocol, with prolonged QT interval and borderline ST elevations in the lateral leads. 

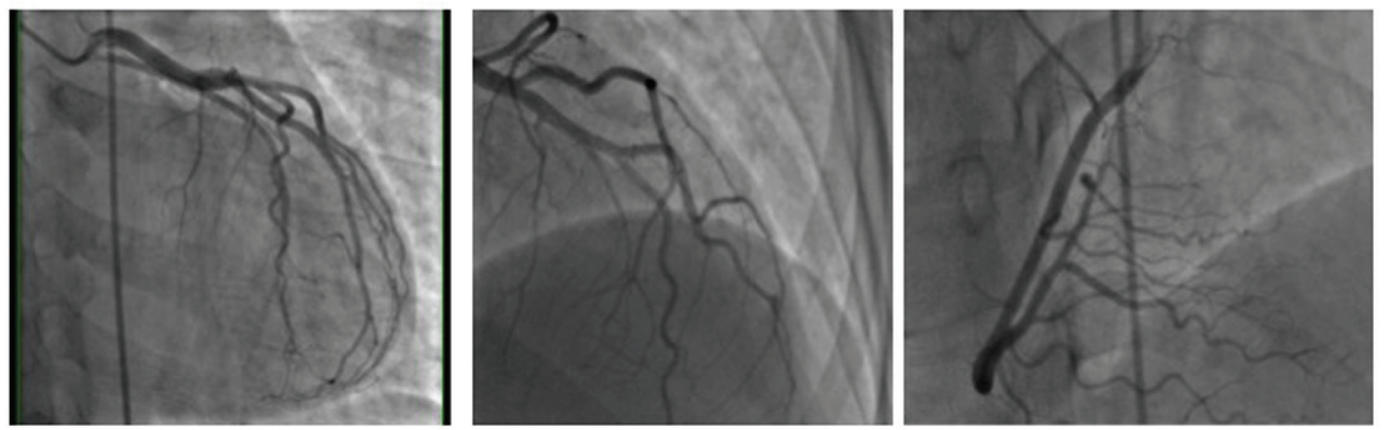

Figure 3. Cardiac catheterization illustrating normal coronary arteries.

served ejection fraction of $50-55 \%$ (Fig. 3).

Although he has a family history of premature coronary artery disease, no significant findings at this time could be appreciated. Electrophysiology consultation was placed and patient was transferred to the appropriate facility for further management with observation and treatment.

\section{Discussion}

The providers of energy drinks state that these drinks contain natural ingredients, which result in increased attention, energy, performance and concentration. In 2011, one study performed by the European Food Safety Authority gathered data from countries within Europe and illustrated that $68 \%$ of adolescents, $30 \%$ of adults, and $18 \%$ of children consumed energy drinks [2]. Few comprehensive reports have clearly linked the many adverse effects reported with its consumption and the specific changes seen in the cardiac workup. These adverse effects may be due to some of the many ingredients found within these beverages, but one of the most attributable ingredients is caffeine. An overconsumption of caffeine can result in palpitations, high blood pressure, central nervous system stimulation, dieresis, vomiting, hypocalcemia, metabolic acidosis, and convulsion [3]. Although caffeine has been found in many beverages such as coffee, it is generally noted to be found in much higher content levels in energy drinks. The amount of caffeine found in energy drinks can range from 80 to $300 \mathrm{mg}$ in a $240 \mathrm{~mL}$ service but serving sizes can vary. Many other ingredients found also include guarana, taurine, glucuronolactone, and $\mathrm{B}$ vitamins, many of which the acute and chronic effects of these combined substances remain an enigma [4]. Guarana, for example, has been shown to have both chronotrope and inotropes and is a plant containing caffeine, theobromine, and theophylline [5].

As our patient did have a borderline QT prolongation, it was unclear at the time of presentation whether it was induced or congenital. The QT was later further prolonged after the initiation of amiodarone, so the question remained whether it was in fact congenital QT prolongation or was a result of other factors. Long QT syndrome is considered a life-threatening arrhythmia and is defined as a delayed myocardial repolarization. This can result in increased risk for torsades des pointes, seizures, and even sudden cardiac death. Itself, it is considered a leading cause of sudden death in otherwise young healthy individuals. The incidence of acquired long QT syndrome is much higher than that of the congenital form. Acquired form is highly associated with cardiac and non-cardiac QT prolonging drugs. Whether this patients QT prolongation was further provoked by the use of amiodarone, it is unclear and remains debatable if his overconsumption of energy drinks also precipitated such events or if there was a previous undocumented condition. In most circumstances cardiac events with long QT syndrome occur during exercise, emotional stress, or at rest [6]. However, it also remains possible that this QT prolongation was further provoked by the hypothermia protocol. It has been shown in previous reports that hypothermia can result in prolongation of PR, QRS, and QTC intervals as well as premature ventricular beats, VT, and VF [7, 8]. However, whether energy drinks can further prolong the QT interval and increase the chances of cardiac issues can still be questioned.

As this patient also has a history of PVCs, it may account for the etiology behind his episode of ventricular fibrillation. However, an association has yet to be properly made. PVCs are relatively common and have an estimated prevalence of $1-4 \%$ in the general population [9]. It occurs when the cardiac electrical activity begins in the purkinje fibers in the ventricles rather than from the sinoatrial node. There are many etiologies associated with PVCs which can in fact be affected by drugs, alcohol, and even caffeine. As this patient has a history of PVCs and has a history of increased red bull consumption, it demonstrates that high levels of energy drinks can not only result in PVCs, but potentially initiate their conversion to ventricular arrhythmias such as ventricular fibrillation in a young patient.

Energy drinks have been shown in the past to result in cardiac arrest. In 2007, a man in Australia suffered a cardiac arrest after consuming seven to eight cans of an energy drink while conducting extensive physical therapy $[2,10]$. Avci et al reported a case in the United States of a 28-year-old male who consumed three energy drinks before a basketball match which resulted in cardiac arrest and death 3 days after $[2,11]$. Unfortunately, the exact etiology has yet to be fully determined. Through this report, we demonstrate one possible mechanism of action in which cardiac arrhythmias such as PVCs can not only develop but progress to ventricular fibrillation and whether there may be an underlying connection between energy drink use and QT prolongation. 


\section{Grant Support}

None.

\section{Financial Disclosures}

None.

\section{Conflict of Interest}

This is to state that there has been no activity or involvements that will raise the question of bias in this case report or any of the conclusions or opinions that it stands for.

\section{References}

1. Zucconi S, Volpato C, Adinolfi F, Gandini E, Gentile E, Loi A, et al. Gathering consumption data on specific consumer groups of energy drinks. Parma: Supporting Publications (2013).

2. Breda JJ, Whiting SH, Encarnacao R, Norberg S, Jones R, Reinap M, Jewell J. Energy drink consumption in europe: a review of the risks, adverse health effects, and policy options to respond. Front Public Health. 2014;2:134.

3. World Health Organization. WHO Basic Analytical Toxi- cology (2005). Available from: http://www.who.int/ipcs/ publications/training_poisons/basic_analytical_tox/en/ index.html.

4. Higgins JP, Tuttle TD, Higgins CL. Energy beverages: content and safety. Mayo Clin Proc. 2010;85(11):10331041.

5. Seifert SM, Schaechter JL, Hershorin ER, Lipshultz SE. Health effects of energy drinks on children, adolescents, and young adults. Pediatrics. 2011;127(3):511-528.

6. Priori SG, Napolitano C, Schwartz P. Genetics of cardiac arrhythmias. Braunwalds's Heart Disease. 8th ed. Philadelphia: Saunders Elsevier; 2007:101-109.

7. Slovis C, Jenkins R. ABC of clinical electrocardiography: Conditions not primarily affecting the heart. BMJ. 2002;324(7349):1320-1323.

8. Khan JN, Prasad N, Glancy JM. QTc prolongation during therapeutic hypothermia: are we giving it the attention it deserves? Europace. 2010;12(2):266-270.

9. Kennedy HL, Whitlock JA, Sprague MK, Kennedy LJ, Buckingham TA, Goldberg RJ. Long-term follow-up of asymptomatic healthy subjects with frequent and complex ventricular ectopy. N Engl J Med. 1985;312(4):193197.

10. Berger AJ, Alford K. Cardiac arrest in a young man following excess consumption of caffeinated "energy drinks". Med J Aust. 2009;190(1):41-43.

11. Avci S, Sarikaya R, Buyukcam F. Death of a young man after overuse of energy drink. Am J Emerg Med. 2013;31(11):1624 e1623-1624. 